\title{
Circular RNA expression profiles of peripheral blood mononuclear cells in rheumatoid arthritis patients, based on microarray chip technology
}

\author{
FENGPING ZHENG, XIANGQI YU, JIAHUANG HUANG and YONG DAI \\ Clinical Medical Research Center, The Second Clinical Medical College of Jinan University, \\ Shenzhen People's Hospital, Shenzhen, Guangdong 518020, P.R. China
}

Received April 26, 2017; Accepted September 7, 2017

DOI: $10.3892 / \mathrm{mmr} .2017 .7638$

\begin{abstract}
Rheumatoid arthritis (RA) is a systemic autoimmune disease characterized by chronic synovial inflammation and finally leads to variable degrees of bone and cartilage erosion. The diagnosis of RA is not an accurate indicator, but a series of scores and the mechanisms underlying it remain only partially understood. The present study explored whether circular RNAs (circRNAs) contribute to the RA pathophysiological mechanism. Total RNA from peripheral blood mononuclear cells of 10 RA patients and 10 healthy controls were extracted and circRNA expression profiling was followed by microarray analysis. In addition, circRNA interactions with microRNAs were performed and microRNA response elements were listed to identify differentially expressed binding site targets in RA. Reverse transcription-quantitative polymerase chain reaction amplification (RT-qPCR) was used to verify the differential expression of circRNAs. A total of 584 circRNAs were differentially expressed in RA patients vs. healthy controls, by circRNA microarray, including 255 circRNAs which were significantly upregulated and 329 downregulated among the RA samples. RT-qPCR validation demonstrated that the expression levels of hsa_circRNA_104194, hsa_circRNA_104593, hsa_circRNA_103334, hsa_circRNA_101407 and hsa_circRNA_102594 were consistent with the results from the microarray analysis. The current study presented differentially expressed circRNAs and their corresponding microRNA binding sites in RA. circRNAs may exhibit a role
\end{abstract}

Correspondence to: Professor Yong Dai, Clinical Medical Research Center, The Second Clinical Medical College of Jinan University, Shenzhen People's Hospital, 1017 Dongmen North Road, Shenzhen, Guangdong 518020, P.R. China

E-mail: daiyong22@aliyun.com

Key words: rheumatoid arthritis, circular RNA, PBMCs, RT-PCR, microRNA in the regulation of expression of symbol genes that influence the occurrence and development of RA.

\section{Introduction}

Rheumatoid arthritis (RA) is a systemic autoimmune disease characterized by chronic synovial inflammation and finally leads to variable degrees of bone and cartilage erosion (1). Presently, the mechanisms and etiology underlying this disease is still not fully understood, besides, there are no satisfactory therapeutic strategies to cure RA $(2,3)$. Patients untreated may suffer short- and long-term joint destruction and functional disability for progressive course including inflammation and antibody attack. The symptoms that allow recognized and diagnosed mainly according to new classification criteria for RA in 2010 developed by The American College of Rheumatology (ACR) and European League Against Rheumatism (EULAR) (4). If the anti-citrullinated antibody (ACPA) and rheumatoid factor (RF) are negative or affected joints are indiscoverable, patients with RA in an earlier phase maybe misdiagnose. And untimely treatment of early RA would lead to a worse clinical outcome (5). Therefore, new and sensitive biomarkers to recognize RA and to give specific treatment as early as possible are necessary for positive therapeutic outcome.

miRNA is one class of the ncRNA and is extensively studied in eukaryotic gene regulation, which plays a significant role in the development of RA (6). Recently, circRNAs can inhibit the function of miRNAs by binding to miRNAs as sponges was reported (7). circRNAs, form as integral and conserved closed continuous loops structures wherein the $3^{\prime}$ and $5^{\prime}$ ends are interconnected, are a large novel class of non-coding RNAs that is highly exist in the eukaryotic transcriptome $(8,9)$. It could resistance to RNase R conferred by its structure of covalently closed loops (10). In the past, its existence was ignored and was regarded as wrong coded (11). However, it was abundant and stable and involves transcriptional and post-transcriptional gene expression regulation (12).

The representative example is mammalian circRNA called CDR1 antisense (CDR1as). CDR1as is a circRNA sponge of miR-7 that provides more than 70 miR-7 seed binding sites, which shows high-frequency interactions 
between circRNAs and miRNAs (13). Recently, there is growing evidence shows that circRNAs can regulate the expression of miRNAs by sponge adsorption, which affects the development of the diseases. circRNAs were found to be important for gastric cancer, colorectal cancer, osteoarthritis and neurodegenerative pathologies (14), which provide a new directions to explore the new diagnosis of these diseases. With the advent of novel biochemical and computational approaches, circRNAs have been becoming a research hotspot in the RNA field (15).

CircRNA has been measured in tissue, serum, exosomes and other body fluids in various diseases $(16,17)$, but the relation between circRNAs and RA remain unclear. Therefore we assessed the differentially expression ofcircRNA in PBMC of RA patients and analyzed the symbol genes that potentially involves pathogenesis and interaction between circRNAs and their paring miRNAs, aiming to explore circRNAs involved in RA and evaluate possible miRNA targets through circRNAs MRE sequence analysis, and provide a novel insight into the circRNAs in PBMC of RA patients in this study.

\section{Materials and methods}

Sample collection. Totally twenty RA patients and healthy people age and gender matched were selected at Xiangmihu People's Hospital, the Fourth People's Hospital of Shenzhen. After selected, blood specimens were collected in March-May in 2014. The experimental study was approved by the hospital ethics committee. Besides, all participants have signed the informed consents form. The PBMC of the blood were immediately separated from 10 paired blood samples, which were obtained from vein of RA patients and HCs.

RNA extraction. The samples were following placed in RNAlater (Qiagen, Hilden, Germany) and stored at $-80^{\circ} \mathrm{C}$. After extraction of total RNA with TRIzol (Thermo Fisher Scientific, Inc., Waltham, MA, USA), the determination of RNA quantity and quality was conducted according to the manufacturer's protocol by NanoDrop ND-1000 (NanoDrop; Thermo Fisher Scientific, Inc., Wilmington, DE, USA). In addition, RNA integrity was assessed by standard denaturing agarose gel electrophoresis.

RNA labeling and microarray hybridization. Sample labeling and array hybridization were performed according to the manufacturer's protocol (Arraystar Inc., Rockville, MD, USA). Briefly, linear RNAs were firstly removed by Rnase R (Epicentre; Illumina, Inc., San Diego, CA, USA). Following, a random priming method (Arraystar Super RNA Labeling kit; Arraystar Inc.) was used to amplify and transcribed each sample into fluorescent cRNA. Then, the labeled cRNAs were purified by RNeasy Mini kit (Qiagen), and concentration and specific activity of it (pmol Cy3/ $\mu \mathrm{g}$ cRNA) were measured by NanoDrop ND-1000. Subsequently, $1 \mu 1$ 25X fragmentation buffer and $1 \mu \mathrm{l} 25 \mathrm{X}$ fragmentation buffer were added to $1 \mu \mathrm{l}$ each labeled cRNA, then the mixture were placed at $60^{\circ} \mathrm{C}$ for $30 \mathrm{~min}$. After, $25 \mu \mathrm{l} 2 \mathrm{X}$ Hybridization buffer was used to dilute the labeled cRNA. The microarray slides were incubated in an Agilent Hybridization Oven at $65^{\circ} \mathrm{C}$ for $17 \mathrm{~h}$. Finally, the hybridized array was fixed and scanned using the Axon
GenePix 4000B microarray scanner (Molecular Devices, Inc., Sunnyvale, CA, USA) after washed.

Data collection. GenePix Pro 6.0 software (Axon; Molecular Devices, LLC, Sunnyvale, CA, USA) was used for raw data extraction and grid alignment from scanned images. The $\mathrm{R}$ software package was used for quantile normalization of raw data and subsequent data processing. After that, the filtering of low intensive substance was performed, and at least $50 \%$ of the circRNAs samples considered flag 'expressed' ( $>2$ times background standard deviation) were conserved for further analyses.

The 'fold-change' of each circRNA was computed to compare the profile differences between the samples of RA patients and healthy controls. t-test was performed to evaluate the statistical significance. P-values $\leq 0.05$ with the 'fold-changes' of circRNA $\geq 2.0$ were considered significantly differentially expressed ones. We filter the analysis outputs and rank the differentially expressed circRNAs according to fold-change and P-value by using Microsoft Excel's Data/Sort and Filter functionalities (Microsoft Corporation, Redmond, WA, USA).

Data analysis and annotation for circRNA/miRNA interaction. We selected the top 10 up- and downregulated circRNAs in PBMCs and plasma for further analysis. PubMed (National Center for Biotechnology Information, Bethesda, MD, USA) was used to analyze differentially expressed circRNAs. circRNA/miRNA interactions were predicted to facilitate our study by using Arraystar miRNA target prediction software based on TargetScan and miRanda. The differentially expressed circRNAs within all the comparisons were annotated in detail with the circRNA/miRNA interaction information. Additionally, we analyzed the sequences of the MREs and the top 10 significantly differentially expressed circRNAs and predicted miRNA targets were also analyzed for their correlation with miRNAs previously described.

Validation of differentially expressed circRNA by reverse transcription-quantitative polymerase chain reation (RT-qPCR). Five circRNAs exhibiting up- or downregulated expression was chosen for results verification by RT-qPCR. The primers are showed in Table I. In order to reduce errors caused by potential variation in concentration and transcription efficiency of RNA, evaluation of the reference gene $\beta$-actin was served as an internal control. Briefly, the cycle parameters for the PCR reaction were $95^{\circ} \mathrm{C}$ for $5 \mathrm{~min}$, followed by 40 amplification cycles of a denaturing step at the same temperature for $10 \mathrm{sec}$ and an annealing/extension step of $60 \mathrm{sec}$ at $60^{\circ} \mathrm{C}$. Following, the amplification product concentration for each sample was generated by Rotor-Gene Real-Time Analysis Software 6.0 (Qiagen). Fold-changes were calculated using the $2^{-\Delta \Delta \mathrm{Cq}}$ method. The relative values of gene expression were taken as the ratio of the sample to the internal control.

\section{Results}

Quality control. RNA quality and quantity were measured using the NanoDrop ND-1000. RNA integrity was successfully tested by standard denaturing agarose gel electrophoresis 
Table I. Reverse transcription-quantitative polymerase chain reaction primer list.

\begin{tabular}{|c|c|c|c|}
\hline Gene & Primer sequence & Annealing temp $\left({ }^{\circ} \mathrm{C}\right)$ & Product size (bp) \\
\hline \multirow[t]{2}{*}{$\beta$-actin $(\mathrm{H})$} & F: 5'-GTGGCCGAGGACTTTGATTG-3' & & \\
\hline & R: 5'-CCTGTAACAACGCATCTCATATT-3' & 60 & 73 \\
\hline hsa_circRNA_104593 & F: 5'-TGCCAGACACATACAAAGATAAGC-3' & & \\
\hline (hsa_circ_0083964) & R: 5'-GGTATCTGGTGGCATCTCATCC-3' & 60 & 160 \\
\hline hsa_circRNA_104194 & F: 5'-GATAAAAACTAACCACCATCTTGC-3' & & \\
\hline (hsa_circ_0004712) & R: 5'-AAGGTAGTCTTCATCCAGCAGG-3' & 60 & 177 \\
\hline hsa_circRNA_103334 & F: 5'-ATTTGGAGTCTGGGAGTGAT-3' & & \\
\hline (hsa_circ_0064996) & R: 5'-ACAGTGTTTTGTTAGTTGCTTCT-3' & 60 & 168 \\
\hline hsa_circRNA_101407 & F: 5'-CAGCACGACAACATTATTGCCTAC-3' & & \\
\hline (hsa_circ_0032683) & R: 5'-ACGACGTTCCTTCTCAGACAGC-3' & 60 & 142 \\
\hline hsa_circRNA_102594 & F: 5'-CACCATCAGCCATAGATCCTC-3' & & \\
\hline (hsa_circ_0052012) & R: 5'-CTTGCCATCCATCCCACATA-3' & 60 & 118 \\
\hline hsa_circRNA_102822 & F: 5'-ACTGCTGGATACTAAATGTGATGC-3' & & \\
\hline (hsa_circ_0056536) & R: 5'-GTTCAAGTTTTCGAGCCTGTTC-3' & 60 & 147 \\
\hline
\end{tabular}

after RNA extraction and prior to sample labeling. For spectrophotometer readings, the O.D. A260/A280 ratio value was established as close to 2.0 for pure RNA (ratios between 1.8 and 2.1 were acceptable), and the O.D.A260/A230 ratio were required to be $>1.8$. For agarose gels, the ribosomal RNA bands of $28 \mathrm{~S}$ and $18 \mathrm{~S}$ were sharp and intensely stained whereas low molecular weight RNAs such as tRNA and 5S ribosomal RNA presented as diffuse bands. DNA contamination of the RNA preparation was evident as a high molecular weight smear or a band migrating above the $28 \mathrm{~S}$ ribosomal RNA band. All quality criteria established by the manufacturer were satisfied for each array to ensure successful microarray analysis.

circRNAs expression profiling in patients with $R A$. In this study, we identified 584 circRNAs that were differentiallyexpressed in RA patients vs. HCs by circRNAs microarray. A total of 255 circRNAs were significantly upregulated and 329 were downregulated among the RA samples (Table II and Fig. 1), the differentiated circRNAs were accounted for fold-change $(\mathrm{FC} \geq 2.0)$ and values $(\leq 0.05)$ in the upregulated and downregulated groups. The box plot view was to show the distributions of expression values between RA and HCs. Moreover, on the basic of all the target circRNA, the 584 differentially expressed circRNAs were converted into a Hierarchical clustering which revealed circRNA expression in normal and RA samples to show distinguishable circRNA expression among these samples (Fig. 2).

Interaction between differential expression of circRNAs and miRNA. With the Arraystar's homemade miRNA target prediction software based on miRanda, microRNAs paring to circRNAs was predicted and the the top 5 predicted miRNA targets of circRNA were selected. The differentially expressed circRNAs is listed with their MREs as supplementary data. The top ten up and downregulated circRNA and their target microRNAs are also shown in the Table II. As we and others have previously observed the differential expression of miRNAs which shown to be associated with physiopathologicmachanisms in RA. All the differentially expressed circRNAs in our study are were notated in detail. For example, in hsa_circRNA_104881, the 101st-106th and 152nd-157th nucleotides are beginning from the 5 'terminal region in the higher expressed gene HSDL2. The nucleotides were completely and represent a complete matched to the seed region of miR-16-5p in the binding mode of Offset 6mer and 7mer-m8 respectively (positions 2-8) (Fig. 3). Through target gene prediction, target genes of the 5 aforementioned miRNAs were collected. The results of Gene symbol analysis on the up and downregulated circRNAs with identified target genes were shown in Table II. Gene symbol analysis revealed that some target genes were involved in some biological processes. These processes were associated with pathogenesis of this disorder.

Validation of circRNAs using RT-qPCR. To verify our observations, hsa_circRNA_104194, hsa_circRNA_104593, hsa_circRNA_103334, hsa_circRNA_101407 and hsa_ circRNA_102594 were selected to be further verifled using RT-qPCR. The results are shown in Fig. 4. For example, the expression of hsa_circRNA_104194 was upregulated 1.64-fold in the RA group compared to the control group $(\mathrm{P}<0.010)$, which was consistent with the microarray results. $\beta$-actin was used as the internal control; The calculated ratios of other genes expression are given in Fig. 4.

\section{Discussion}

circRNAs are ubiquitous and widely expressed, shows it is functionally important. The most well known circRNAs is CDR1as, located in cytoplasm (12), and owned 74 miR-7 seed binding sites. It can effectively reduce exerted miR-7 by the function as miRNA sponge in body tissuse and affect disease such as cancers (18). circular Sry is also a demonstration of miRNA sponge (7) and 16 miRNA-138 target sites were found in circular Sry. Besides, there are evidences shows the decrease 


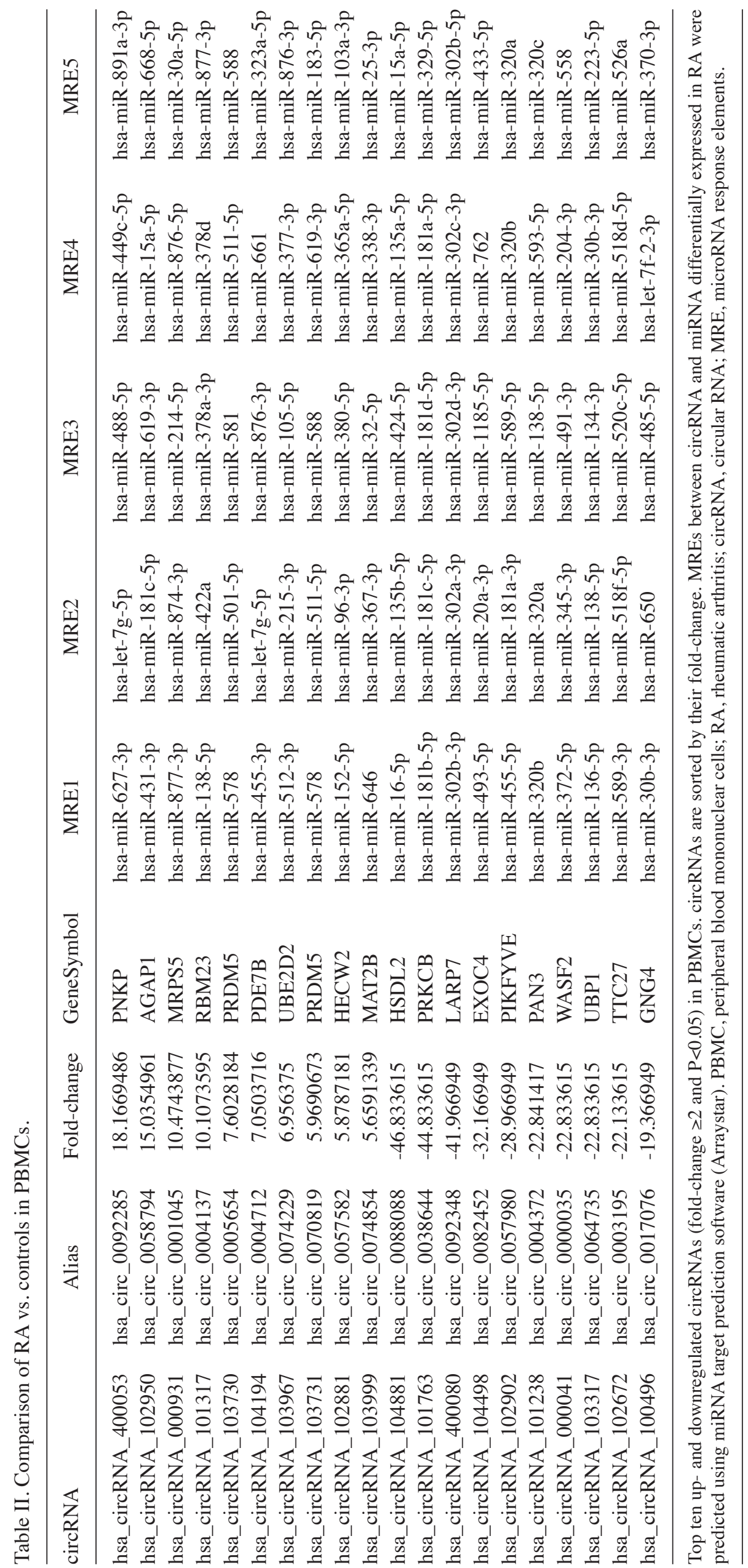




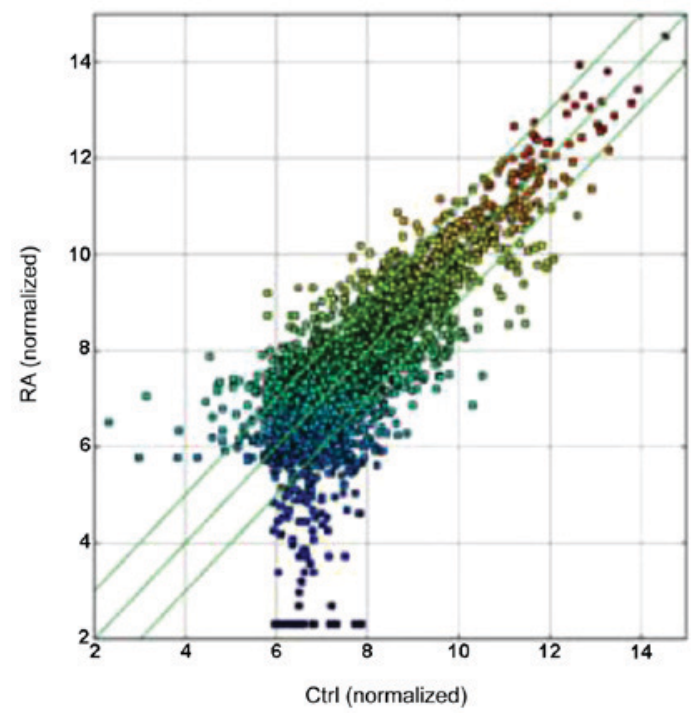

Figure 1. Scatter-plot of circRNA expression variation and reproducibility between RA and control samples. The values of $\mathrm{X}$ and $\mathrm{Y}$ axes in the scatter-plot represent the normalized signal values of the samples $(\log 2$ scaled). The green lines illustrate fold-change. The circRNAs above the top green line and below the bottom green line exhibit $>2.0$ fold-change between RA and controls.

in polymorphisms at miRNA target sites in circRNAs $(19,20)$. circRNAs could potentially regulate mechanism of pathology in diseases.

Although lack of 5'cap and 3'poly (A) tail, circRNA have protein-coding sequences, cap-independent translation of certain linear mRNA and open reading frames, which illustrated that circRNA have potential to translation $(21,22)$, However, no evident was supported the existence of translatable circRNAs (23).

The most in-depth study of the relationship between circRNAs and diseases is cancer. Researchers found their potential of biomarkers to diagnose tumors. For example, cir-ITCH downregulated in esophageal squamous cell carcinoma and colorectal cancer by increasing ITCH expression by suppressing the Wnt/ $\beta$-catenin pathway $(15,24)$. Decreasing Has_circ_002059 can be consider as biomarker in gastric cancer tissues (25) and Has_-circ_0001649 low expressed in hepatocellular carcinoma (HCC) tissues (26). CircRNAs has abundant miRNA attachment sites and more than 800 miRNAs regulate one-third of the genes, especially immune-related genes (27). CircRNAs have high degree of stability in mammalian cells that we speculate that its change can really reflect relationship with the disease compared with other noncoding RNA.

We revealed that circRNAs were differentially expressed in PBMC of RA patients compared with healthy controls in this study. The maximum ratio of up and downregulated is 18.17 and 46.83. Several cicRNAs aberrant distinctly, these circRNAs may play an important role in the pathogenesis of RA.

RA is immune system disorders involved in a variety of factors of immune system disorders. It affects the activities of the joints as well as the functions of many other organs. Although the treatment and diagnosis of RA was improved, the specific mechanism of etiology is still remains unclear. The function circRNAs of in disorders gives us a new direction to understand the RA. The circRNAs can regulate the

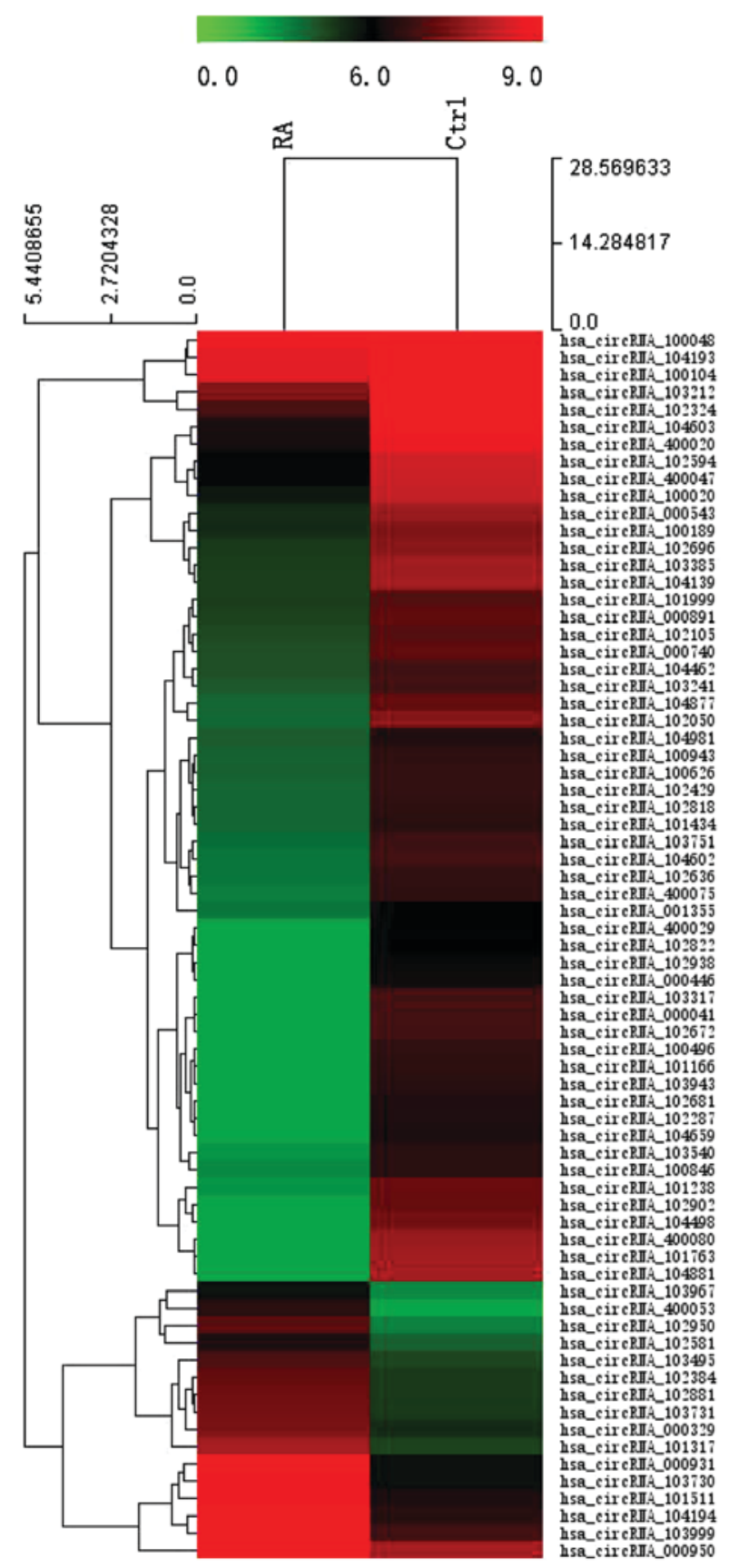

Figure 2. Hierarchical clustering to distinguish differentially expressed circRNAs between RA and control samples. Each column represents one sample and each row represents one circRNA. The dendrogram shows the relationships among the sample expression levels. The top dendrogram separates the expression profiles of the control group from the RA group. Hierarchical cluster analysis results suggest that the difference spectrum is reliable.

expression of the mRNA by reducing the inhibitory effect of miRNAs (28).

In this study, we found that hsa_circ_0092285, hsa circ_0058794 were significantly higher in PBMC of RA patients and hsa_circ_0088088, hsa_circ_0038644 were lower.

hsa_circ_0092285 is aligned from the PNKP gene. Polynucleotide phosphatase/kinase in humans is encoded by the PNKP gene (29). Oxidative stress-induced free radicals can be used as oxidants and inflammatory mediators involving 


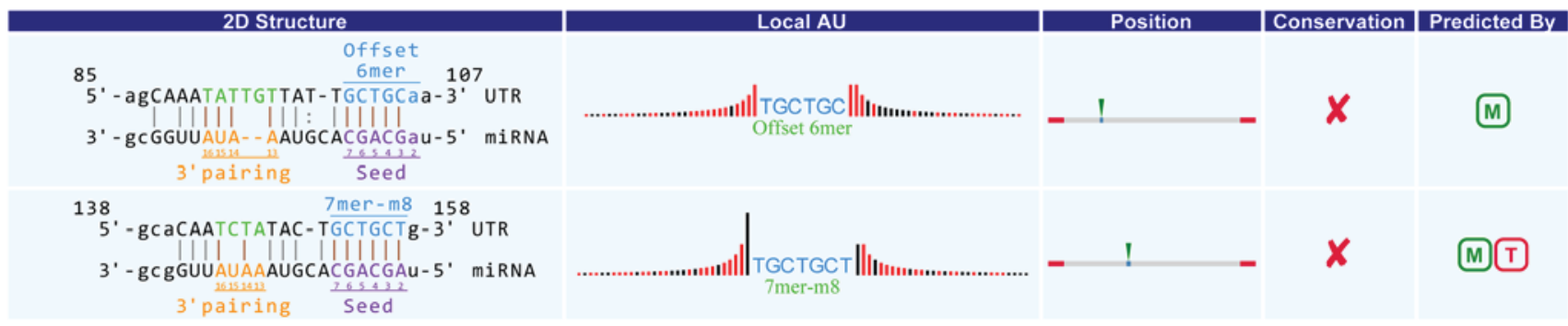

Figure 3. Detailed annotation for circRNA-miRNA interactions. Representative notation example showing the complementary situation of hsa_circRNA_104881 and miR-16-5p. circRNA, circular RNA.
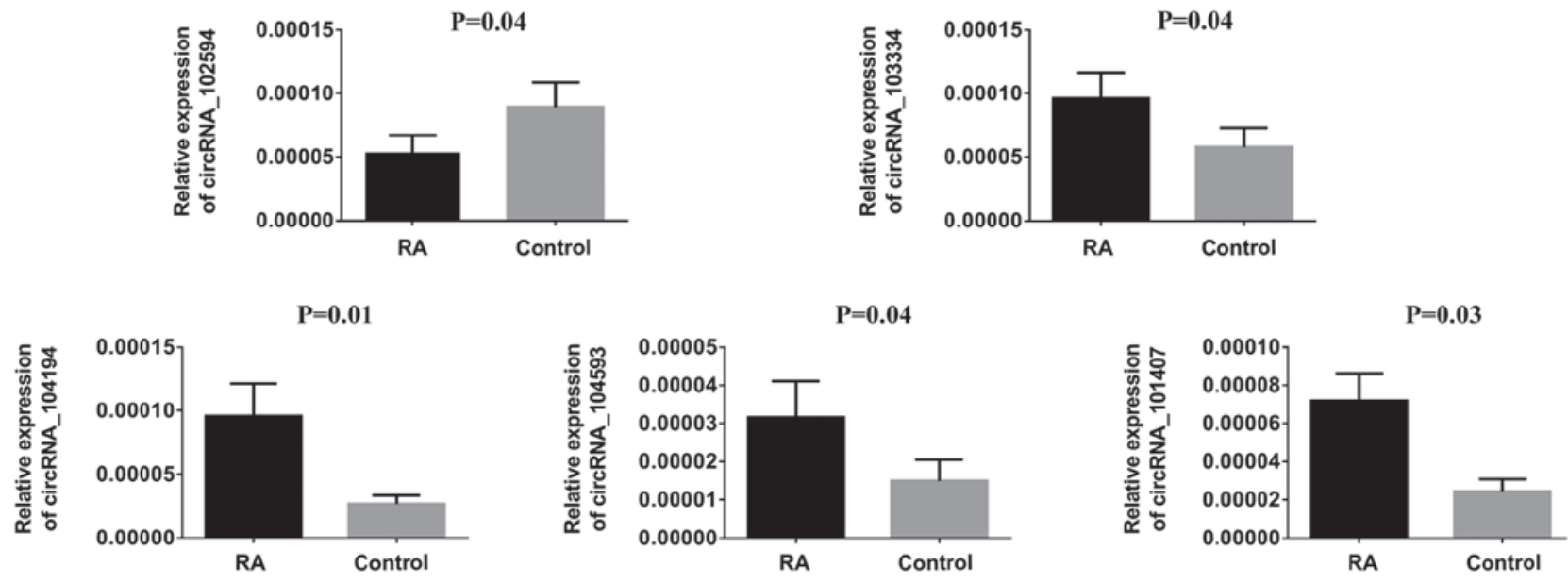

Figure 4. RT-qPCR validation of circRNA. hsa_circRNA_104194, hsa_circRNA_104593, hsa_circRNA_103334, hsa_circRNA_101407 and hsa_ circRNA_102594 were shown significance expression in RA compared to Control. RT-qPCR, reverse transcription-quantitative polymerase chain reaction.

in the pathogenesis of RA $(30,31)$. Polynucleotide phosphatase/kinase plays a major role in the restoration of correct DNA termini following strand cleavage by ROS (32). which induces mutations in itochondrial DNA (mtDNA) lesions (33) and sensitizes cells to hydrogen peroxide (34). Downregulated PNKP gene are the major source of accumulation of strand breaks in mtDNA of hydrogen peroxide-treated cells and H2O2-induced cells (35). Therefore, we hypothesized that PNKP is associated with the development of oxidative stress and inflammation in RA.

hsa_circ_0058794 is spliced from the AGAP1 gene.AGAP1 is a prototype of the ArfGAP protein with the GTPase-like domain and a phosphoinositide-dependent Arf GAP and affects the dynamics of the actin cytoskeleton (36). One of the characteristics of RA synoviocytes is the invasion and migration of cartilage (37). Migrating to the unaffected joints is a manifestation of disease progression. Invasion and migration require the integration of signals from the extracellular environment and remodeling the actin cytoskeleton $(38,39)$. AGAP1 could induce and localizes to Rab4/AP1 containing a putative endosome structure and specifically altering the stress fiber. AGAP1 is a possible link between endocytic traffic anthe actin cytoskeleton (36).

hsa_circ_0088088 is the most pronounced circRNA downregulated in RA and spliced from hydroxysteroid dehydrogenase-like 2 genes. HSDL2 is one of the members of short-chain dehydrogenases/reductase (SDR) superfamily containing a peroxisomal targeting signal (40). Human HSDL2 was found in peroxisomes or mitochondria, some researches assumed the involvement of HSDL2 in fatty acid and cholesterol metabolism and homeostasis for its localization (41). Existence of dyslipidemia in the early RA and RA progression has been confirmed (42). Cardiovascular risk is the main cause of early death in patients with RA (43). Inflammatory cytokines promote lipid breakdown and increase free fatty acid levels, causing endothelial dysfunction, thereby increasing the risk of atherosclerosis in RA (44).

hsa_circ_0038644 is encoded within PRKCB.PRKCB is indicated as a candidate gene associated with the LPS immune response, which involves B cell viability and antigen response (45). Lipopolysaccharide (LPS) is an inflammatory factor, which is a common ligand for TLR2 and TLR4. When LPS binds to TLR2 and TLR4, it activates TLR signal transduction pathway and participates in the immune regulation function (46). Immune disorder is the main pathogenesis of RA. TLRs and LPS considered members of the innate immune system inducing proinflammatory cytokine production has an important role in this disorder. However, TLRs, especially TLR2 and TLR4, play an important role in the pathogenesis of RA (47). PRKCB gene could recruit IKK into lipid rafts and is involved in $\mathrm{B}$-cell receptor (BCR)-mediated NF- $\kappa B$ activation (48).

miRNAs are tissue-specific and stage-specific of disease progression. It involves cancer, cell apoptosis, inflammation, even autoimmune arthritis (49). miRNAs participates in generation of MMPs, leukocyte activation, inflammatory 
responses, and cell proliferation of synoviocytes in rheumatoid joints $(50,51)$. Previous studies have found that many miRNAs were associated with RA.miR-146a, miR-155, miR-132, and miR-16 are increased in PBMCs in RA patients compared to HCs (52).

Recently, miR-24-3p, and miR125a-5p were reported to be helpful in the diagnosis of RA (53) and generation of chemokine and inflammatory cytokines. Many studies have shown that some potential effects between cicrRNAson miRNAs, however, the role of circRNAs and miRNAs on the impaction of RA is not very clear. Theoretically, as a sponge of miRNAs, the circRNA can competitively bind and inhibit the corresponding miRNAs, increasing the target of the corresponding miRNA. hsa_circ_0057980 is significantly downregulated in this study. miR-181d is one of its MREs. Our findings are consistent with a recent study in which miR-181d is significantly increased in RA patients compared with HCs (54). Likewise, hsa-miR-16-5p, one of the hsa_circ_0088088 downregulated in our study with the largest times, means increase distinctly. miR-16 is mainly correlated with the degree of activity of the disease such erythrocyte sedimentation rate (ESR), C-reactive protein (CRP) value, tender joint count (TJC) and TNF- $\alpha$ in RA patients (52). It also parallel to DAS28 index of RA (55). Therefore, we could conclude that miR-16 paly important role in pathogenesis of RA. On the contrary, miR-30a was reported to have dropped significantly in RA, which has a consistent result with our research that hsa_circ_0001045 is significantly upregulated. Low levels of miR-30a have been shown to be associated with reduced cell apoptosis in RA synovialis (56).

Although many miRNAs above have relation with RA described by other researchers, there are still many of them are not clear or not studied so far. Some of them may have certain correlation with the occurrence and development of RA. Studies have found that hsa-let-7g-5p is significantly higher in patients by affecting many metabolic pathways, such as lipid metabolism (57). miR-488 was significantly decreased in osteoarthritis (OA) chondrocytes by regulating zinc transporter SLC39A8/ZIP8. Notably, OA involves musculoskeletal disorder and also leads to joint injury (58).

Even though we have analyzed and discussed the role of circRNA and miRNA in RA, there are also some limitations in this study. First of all, the sample size is too small to get an affirmative conclusion. Secondly, all specimens come from one hospital, resulting in the possibility of regional characteristics. Thirdly, detailed functional analysis is insufficient, so further research needs to improve this deficiency. Maybe, we could investigate the circRNA profile of regulatory $\mathrm{T}$ cells or some other cell kinds which have been demonstrated to play key roles in RA instead of peripheral blood mononuclear cells. Although our study did not select and validate a specific circRNA for the pathophysiology and molecular mechanisms of RA, nevertheless our study may provide a new perspective for the early diagnosis and pathogenesis of RA.

Overall, our study indicated that the expression of circRNAs is significantly different between RA patients and HCs. The circRNA as a miRNA sponge may play an important functional role in RA. We should make good use of their roles in sponging miRNA to prevent the harms caused by these overexpressed or low expressed miRNAs in RA patients for treatment. There is no doubt that the deep study is needed to reveal the role of molecular mechanism of circRNA in the development and progression of RA.

\section{Acknowledgements}

This study was supported by Shenzhen Science and Technology ProjectorofGuangdongProvince(no.JCYJ20160422164313440). We thank all subjects who participated in this study. We also thank the Department of Nephrology, Shenzhen Key Subject of Medicine of Guangdong Province.

\section{References}

1. Firestein GS: Evolving concepts of rheumatoid arthritis. Nature 423: 356-361, 2003.

2. Salemi S, Biondo MI, Fiorentino C, Argento G, Paolantonio M, Di Murro C, Malagnino VA, Canzoni M, Diamanti AP and D'Amelio R: Could early rheumatoid arthritis resolve after periodontitis treatment only?: Case report and review of the literature. Medicine (Baltimore) 93: e195, 2014.

3. Ursini F, Russo E, Letizia Hribal M, Mauro D, Savarino F, Bruno C, Tripolino C, Rubino M, Naty S and Grembiale RD: Abatacept improves whole-body insulin sensitivity in rheumatoid arthritis: An observational study. Medicine (Baltimore) 94: e888, 2015.

4. Aletaha D, Neogi T, Silman AJ, Funovits J, Felson DT, Bingham CO III, Birnbaum NS, Burmester GR, Bykerk VP, Cohen MD, et al: 2010 Rheumatoid arthritis classification criteria: An American College of Rheumatology/European League Against Rheumatism collaborative initiative. Arthritis Rheum 62: 2569-2581, 2010.

5. Goekoop-Ruiterman YP, de Vries-Bouwstra JK, Allaart CF, van Zeben D, Kerstens PJ, Hazes JM, Zwinderman AH, Ronday HK, Han KH, Westedt ML, et al: Clinical and radiographic outcomes of four different treatment strategies in patients with early rheumatoid arthritis (the BeSt study): A randomized, controlled trial. Arthritis Rheum 52: 3381-3390, 2005.

6. Chen XM, Huang QC, Yang SL, Chu YL, Yan YH, Han L, Huang Y and Huang RY: Role of Micro RNAs in the pathogenesis of rheumatoid arthritis: Novel perspectives based on review of the literature. Medicine (Baltimore) 94: e1326, 2015.

7. Hansen TB, Jensen TI, Clausen BH, Bramsen JB, Finsen B, Damgaard CK and Kjems J: Natural RNA circles function as efficient microRNA sponges. Nature 495: 384-388, 2013.

8. Qu S, Yang X, Li X, Wang J, Gao Y, Shang R, Sun W, Dou K and Li H: Circular RNA: A new star of noncoding RNAs. Cancer Lett 365: 141-148, 2015.

9. Conn SJ, Pillman KA, Toubia J, Conn VM, Salmanidis M, Phillips CA, Roslan S, Schreiber AW, Gregory PA and Goodall GJ: The RNA binding protein quaking regulates formation of circRNAs. Cell 160: 1125-1134, 2015.

10. Qu S, Song W, Yang X, Wang J, Zhang R, Zhang Z, Zhang H and $\mathrm{Li} \mathrm{H}$ : Microarray expression profile of circular RNAs in human pancreatic ductal adenocarcinoma. Genom Data 5: 385-387, 2015.

11. Sanger HL, Klotz G, Riesner D, Gross HJ and Kleinschmidt AK: Viroids are single-stranded covalently closed circular RNA molecules existing as highly base-paired rod-like structures. Proc Natl Acad Sci USA 73: 3852-3856, 1976.

12. Memczak S, Jens M, Elefsinioti A, Torti F, Krueger J, Rybak A, Maier L, Mackowiak SD, Gregersen LH, Munschauer M, et al: Circular RNAs are a large class of animal RNAs with regulatory potency. Nature 495: 333-338, 2013.

13. Hansen TB, Kjems J and Damgaard CK: Circular RNA and miR-7 in cancer. Cancer Res 73: 5609-5612, 2013.

14. Kulcheski FR, Christoff AP and Margis R: Circular RNAs are miRNA sponges and can be used as a new class of biomarker. J Biotechnol 238: 42-51,2016.

15. Li F, Zhang L, Li W, Deng J, Zheng J, An M, Lu J and Zhou Y: Circular RNA ITCH has inhibitory effect on ESCC by suppressing the Wnt/ß-catenin pathway. Oncotarget 6: 6001-6013, 2015.

16. Nagpal JK, Dasgupta S, Jadallah S, Chae YK, Ratovitski EA, Toubaji A, Netto GJ, Eagle T, Nissan A, Sidransky D and Trink B: Profiling the expression pattern of GPI transamidase complex subunits in human cancer. Mod Pathol 21: 979-991, 2008. 
17. Mendell JT, ap Rhys CM and Dietz HC: Separable roles for rent $1 / \mathrm{hUpf} 1$ in altered splicing and decay of nonsense transcripts. Science 298: 419-422, 2002.

18. Peng L, Yuan XQ and Li GC: The emerging landscape of circular RNA ciRS-7 in cancer (Review). Oncol Rep 33: 2669-2674, 2015

19. Thomas LF and Saetrom P: Circular RNAs are depleted of polymorphisms at microRNA binding sites. Bioinformatics 30 2243-2246, 2014.

20. Wang Y and Wang Z: Efficient backsplicing produces translatable circular mRNAs. RNA 21: 172-179, 2015.

21. Gilbert WV: Alternative ways to think about cellular internal ribosome entry. J Biol Chem 285: 29033-29038, 2010.

22. Guo JU, Agarwal V, Guo H and Bartel DP: Expanded identification and characterization of mammalian circular RNAs. Genome Biol 15: 409, 2014

23. Jeck WR, Sorrentino JA, Wang K, Slevin MK, Burd CE, Liu J, Marzluff WF and Sharpless NE: Circular RNAs are abundant, conserved, and associated with ALU repeats. RNA 19: 141-157, 2013.

24. Huang G, Zhu H, Shi Y, Wu W, Cai H and Chen X: cir-ITCH plays an inhibitory role in colorectal cancer by regulating the Wnt/ $\beta$-catenin pathway. PLoS One 10: e0131225, 2015.

25. Zhao ZJ and Shen J: Circular RNA participates in the carcinogenesis and the malignant behavior of cancer. RNA Biol 14 514-521, 2017.

26. Qin M, Liu G, Huo X, Tao X, Sun X, Ge Z, Yang J, Fan J, Liu L and Qin W: Hsa_circ_0001649: A circular RNA and potential novel biomarker for hepatocellular carcinoma. Cancer Biomark 16: 161-169, 2016.

27. Bentwich I, Avniel A, Karov Y, Aharonov R, Gilad S, Barad O, Barzilai A, Einat P, Einav U, Meiri E, et al: Identification of hundreds of conserved and nonconserved human microRNAs. Nat Genet 37: 766-770, 2005

28. Cheng DL, Xiang YY, Ji LJ and Lu XJ: Competing endogenous RNA interplay in cancer: Mechanism, methodology, and perspectives. Tumour Biol 36: 479-488, 2015.

29. Weinfeld M, Mani RS, Abdou I, Aceytuno RD and Glover JN: Tidying up loose ends: The role of polynucleotide kinase/phosphatase in DNA strand break repair. Trends Biochem Sci 36 262-271, 2011.

30. Hitchon CA and El-Gabalawy HS: Oxidation in rheumatoid arthritis. Arthritis Res Ther 6: 265-278, 2004.

31. Phaniendra A, Jestadi DB and Periyasamy L: Free radicals: Properties, sources, targets, and their implication in various diseases. Indian J Clin Biochem 30: 11-26, 2015.

32. Das A, Wiederhold L, Leppard JB, Kedar P, Prasad R, Wang H, Boldogh I, Karimi-Busheri F, Weinfeld M, Tomkinson AE, et al: NEIL2-initiated, APE-independent repair of oxidized bases in DNA: Evidence for a repair complex in human cells. DNA Repair (Amst) 5: 1439-1448, 2006.

33. Ralph SJ, Rodriguez-Enriquez S, Neuzil J, Saavedra E and Moreno-Sánchez R: The causes of cancer revisited: 'Mitochondrial malignancy' and ROS-induced oncogenic transformation-why mitochondria are targets for cancer therapy. Mol Aspects Med 31: 145-170, 2010.

34. Rasouli-Nia A, Karimi-Busheri F and Weinfeld M: Stable down-regulation of human polynucleotide kinase enhances spontaneous mutation frequency and sensitizes cells to genotoxic agents. Proc Natl Acad Sci USA 101: 6905-6910, 2004.

35. Tahbaz N, Subedi S and Weinfeld M: Role of polynucleotide kinase/phosphatase in mitochondrial DNA repair. Nucleic Acids Res 40: 3484-3495, 2012

36. Nie Z, Stanley KT, Stauffer S, Jacques KM, Hirsch DS, Takei J and Randazzo PA: AGAP1, an endosome-associated, phosphoinositide-dependent ADP-ribosylation factor GTPase-activating protein that affects actin cytoskeleton. J Biol Chem 277: 48965-48975, 2002.

37. Bartok B, Hammaker D and Firestein GS: Phosphoinositide 3 -kinase $\delta$ regulates migration and invasion of synoviocytes in rheumatoid arthritis. J Immunol 192: 2063-2070, 2014.

38. Ridley AJ, Schwartz MA, Burridge K, Firtel RA, Ginsberg MH, Borisy G, Parsons JT and Horwitz AR: Cell migration: Integrating signals from front to back. Science 302: 1704-1709, 2003.
39. Parsons JT, Horwitz AR and Schwartz MA: Cell adhesion: Integrating cytoskeletal dynamics and cellular tension. Nat Rev Mol Cell Biol 11: 633-643, 2010

40. Dai J, Xie Y, Wu Q, Wang L, Yin G, Ye X, Zeng L, Xu J, Ji C, $\mathrm{Gu}$ S, et al: Molecular cloning and characterization of a novel human hydroxysteroid dehydrogenase-like 2 (HSDL2) cDNA from fetal brain. Biochem Genet 41: 165-174, 2003.

41. Skogsberg J,Lundström J, Kovacs A, Nilsson R, Noori P, Maleki S, Köhler M, Hamsten A, Tegnér J and Björkegren J: Transcriptional profiling uncovers a network of cholesterol-responsive atherosclerosis target genes. PLoS Genet 4: e1000036, 2008.

42. Peters MJ, Vis M, van Halm VP, Wolbink GJ, Voskuyl AE, Lems WF, Dijkmans BA, Twisk JW, de Koning MH, van de Stadt RJ and Nurmohamed MT: Changes in lipid profile during infliximab and corticosteroid treatment in rheumatoid arthritis. Ann Rheum Dis 66: 958-961, 2007.

43. Pincus T, Sokka T and Wolfe F: Premature mortality in patients with rheumatoid arthritis: Evolving concepts. Arthritis Rheum 44: 1234-1236, 2001

44. Boden G: Obesity and free fatty acids. Endocrinol Metab Clin North Am 37: 635-646, viii-ix, 2008.

45. Siwek M, Slawinska A, Rydzanicz M, Wesoly J, Fraszczak M, Suchocki T, Skiba J, Skiba K and Szyda J: Identification of candidate genes and mutations in QTL regions for immune responses in chicken. Anim Genet 46: 247-254, 2015.

46. Gierut A, Perlman H and Pope RM: Innate immunity and rheumatoid arthritis. Rheum Dis Clin North Am 36: 271-296, 2010.

47. Roelofs MF, Wenink MH, Brentano F, Abdollahi-Roodsaz S, Oppers-Walgreen B, Barrera P, van Riel PL, Joosten LA, Kyburz D, van den Berg WB and Radstake TR: Type I interferons might form the link between Toll-like receptor (TLR) 3/7 and TLR4-mediated synovial inflammation in rheumatoid arthritis (RA). Ann Rheum Dis 68: 1486-1493, 2009.

48. Sheng YJ, Gao JP, Li J, Han JW, Xu Q, Hu WL, Pan TM, Cheng YL, Yu ZY, Ni C, et al: Follow-up study identifies two novel susceptibility loci PRKCB and 8p11.21 for systemic lupus erythematosus. Rheumatology (Oxford) 50: 682-688, 2011.

49. Nagata Y, Nakasa T, Mochizuki Y, Ishikawa M, Miyaki S, Shibuya H, Yamasaki K, Adachi N, Asahara H and Ochi M: Induction of apoptosis in the synovium of mice with autoantibody-mediated arthritis by the intraarticular injection of double-stranded MicroRNA-15a. Arthritis Rheum 60: 2677-2683, 2009.

50. Nakasa T, Nagata Y, Yamasaki K and Ochi M: A mini-review: microRNA in arthritis. Physiol Genomics 43: 566-570, 2011.

51. Baxter D, McInnes IB and Kurowska-Stolarska M: Novel regulatory mechanisms in inflammatory arthritis: A role for microRNA. Immunol Cell Biol 90: 288-292, 2012.

52. Pauley KM, Satoh M, Chan AL, Bubb MR, Reeves WH and Chan EK: Upregulated miR-146a expression in peripheral blood mononuclear cells from rheumatoid arthritis patients. Arthritis Res Ther 10: R101, 2008.

53. Liu Y, Dong J, Mu R, Gao Y, Tan X, Li Y, Li Z and Yang G: MicroRNA-30a promotes B cell hyperactivity in patients with systemic lupus erythematosus by direct interaction with Lyn. Arthritis Rheum 65: 1603-1611, 2013.

54. Wang W, Zhang Y, Zhu B, Duan T, Xu Q, Wang R, Lu L and Jiao Z: Plasma microRNA expression profiles in Chinese patients with rheumatoid arthritis. Oncotarget 6: 42557-42568, 2015.

55. Murata K, Yoshitomi H, Tanida S, Ishikawa M, Nishitani K, Ito $\mathrm{H}$ and Nakamura T: Plasma and synovial fluid microRNAs as potential biomarkers of rheumatoid arthritis and osteoarthritis. Arthritis Res Ther 12: R86, 2010.

56. Xu K, Xu P, Yao JF, Zhang YG, Hou WK and Lu SM: Reduced apoptosis correlates with enhanced autophagy in synovial tissues of rheumatoid arthritis. Inflamm Res 62: 229-237, 2013.

57. Zafari S, Backes C, Meese E and Keller A: Circulating biomarker panels in Alzheimer's disease. Gerontology 61: 497-503, 2015.

58. Egloff $\mathrm{C}$, Hugle $\mathrm{T}$ and Valderrabano V: Biomechanics and pathomechanisms of osteoarthritis. Swiss Med Wkly 142: w13583, 2012. 